\title{
Presence of cutaneous lesion is a poor prognostic factor in patients with smoldering-type adult T-cell leukemia-lymphoma
}

\author{
Kentaro Yonekura ${ }^{1,2^{*}}$, Atae Utsunomiya ${ }^{3}$, Kazuhiro Kawai ${ }^{1}$, Yoshifusa Takatsuka ${ }^{3}$, Shogo Takeuchi ${ }^{3}$, \\ Masahito Tokunaga ${ }^{3}$, Ayumu Kubota ${ }^{3}$, Tamotsu Kanzaki $^{2}$, Youhei Uchida ${ }^{1}$, Takuro Kanekura ${ }^{1}$ \\ From 15th International Conference on Human Retroviruses: HTLV and Related Viruses \\ Leuven and Gembloux, Belgium. 5-8 June 2011
}

\section{Background}

Prognosis of indolent types of adult T-cell leukemialymphoma (ATL) including the smoldering type was recently reported to be poorer than that shown in previous studies. Prognostic factors of smoldering-type ATL have not been defined. Cutaneous-type, which is defined as cases of smoldering type predominantly involve skin with or without peripheral blood involvement has been proposed as a distinct clinical subtype. Prognosis of cutaneous-type ATL was reported to be poorer than that of smoldering-type without cutaneous involvement.

\section{Aim}

To determine prognostic factors for survival and disease progression of smoldering-type ATL.

\section{Patients}

Thirty-one patients with smoldering-type ATL including 21 with cutaneous lesion.

\section{Methods}

Multivariate Cox proportional hazards model was used to identify variables associated with survival and disease progression. Peripheral blood abnormal lymphocytes ( $<5 \%$ vs $\geq 5 \%$ ), serum lactate dehydrogenase (normal vs high), albumin level ( $<4 \mathrm{vs} \geq 4 \mathrm{~g} / \mathrm{dl})$, and cutaneous lesion (none vs present) were used as variables. Overall survival (OS) and progression-free survival (PFS) were

\footnotetext{
* Correspondence: orch@m.kufm.kagoshima-u.ac.jp ${ }^{1}$ Department of Dermatology, Kagoshima University Graduate School of Medical and Dental Sciences, Kagoshima, Kagoshima, 890-8520, Japan Full list of author information is available at the end of the article
}

estimated using the Kaplan-Meier method and compared using the log-rank test.

\section{Results}

In the multivariate analysis, presence of cutaneous lesion was the significant prognostic factor for PFS (hazard ratio, 8.69; 95\% confidence interval, 1.4-54.0; $\mathrm{P}=0.02$ ). Peripheral blood abnormal lymphocytes, serum lactate dehydrogenase, or albumin level was not significant. None of the variables was significantly associated with OS in the multivariate analysis. OS $(\mathrm{P}=0.117)$ and PFS $(\mathrm{P}=0.089)$ of the patients with cutaneous lesion were worse as compared to those of the patients without cutaneous lesion, though statistically not significant.

\section{Conclusions}

In this study we confirmed that presence of cutaneous lesion is an independent prognostic factor in patients with smoldering-type ATL. Cases of smoldering-type with cutaneous lesion should be classified as cutaneous-type ATL irrespective of the peripheral blood involvement.

\section{Author details \\ 'Department of Dermatology, Kagoshima University Graduate School of Medical and Dental Sciences, Kagoshima, Kagoshima, 890-8520, Japan. \\ ${ }^{2}$ Department of Dermatology, Imamura Bun-in Hospital, Kagoshima, Kagoshima, 890-0064, Japan. ${ }^{3}$ Department of Hematology, Imamura Bun-in Hospital, Kagoshima, Kagoshima, 890-0064, Japan.}

Published: 6 June 2011
doi:10.1186/1742-4690-8-S1-A35

Cite this article as: Yonekura et al:: Presence of cutaneous lesion is a poor prognostic factor in patients with smoldering-type adult T-cell leukemia-lymphoma. Retrovirology 2011 8(Suppl 1):A35.

\section{C)

